\title{
Intertwined internal rays in Julia sets of rational maps
}

\author{
by
}

\author{
Robert L. Devaney (Boston, MA)
}

\begin{abstract}
We show how the well-known concept of external rays in polynomial dynamics may be extended throughout the Julia set of certain rational maps. These new types of rays, which we call internal rays, meet the Julia set in a Cantor set of points, and each of these rays crosses infinitely many other internal rays at many points. We then use this construction to show that there are infinitely many disjoint copies of the Mandelbrot set in the parameter planes for these maps.
\end{abstract}

External rays in both the dynamical and parameter planes provide an extremely important tool in complex dynamics. External rays arise as follows. For complex polynomials, there is always a basin of attraction of the fixed point at $\infty$. If the polynomial has degree $n$, it is well known that, in a neighborhood of $\infty$, the polynomial is analytically conjugate to the map $z \mapsto z^{n}$ also defined in a neighborhood of infinity (the so-called Böttcher coordinate). Now $z^{n}$ maps straight rays of the form $t \exp (2 \pi i \theta)$ for $t>1$ to straight rays of the form $t^{n} \exp (2 \pi i n \theta)$. So, under the conjugacy, the images of these straight rays in the basin of $\infty$ are also preserved by the polynomial. These images are the external rays for the polynomial. How these rays "land" on the Julia set often completely determines the structure of the Julia set for this map.

In this paper, we shall concentrate on rational maps, not polynomials. Specifically, we consider the family of maps

$$
F_{\lambda}(z)=z^{n}+\frac{\lambda}{z^{n}}
$$

where $\lambda \in \mathbb{C}$ and $n>2$. We are interested in this family for two reasons. First, like polynomials, these maps always have a superattracting fixed point at $\infty$, so we have external rays for these maps. Second, like the family $z^{n}+c$, $n>1$, there is essentially only one critical orbit for each member of this family, so the natural parameter plane is the $\lambda$-plane. 
Our goal in this paper is to introduce a different type of ray into the dynamical planes of these maps, namely, internal rays. Basically, internal rays in the dynamical plane are curves that connect the endpoints of external rays to the origin (the only pole) by winding in a specific way through the Julia set. Unlike the external rays, however, the internal rays always cross other internal rays, usually at multiple points; hence they are "intertwined." In this paper, we will describe a special Cantor set of such internal rays. These rays are important in that how they wind through the Julia sets of these maps is the same no matter what the parameter $\lambda$ is; for other internal rays, how they wind through the Julia set does depend upon the parameter. Moreover, how these special internal rays cross each other subdivides the Julia sets into regions where the structure is then easy to understand. As an example, we shall use the structure of the internal rays in the dynamical planes for these maps to prove the following result:

THEOREM. In the parameter plane for $z^{n}+\lambda / z^{n}, n>2$, there are countably many distinct copies of the Mandelbrot set, i.e., Mandelbrot sets that are not subsets of larger Mandelbrot sets. In particular, there are $(n-2)$. $(2 n-2)^{k-1}$ distinct baby Mandelbrot sets with base period $k$ in the parameter plane for $F_{\lambda}$.

In a subsequent paper [4] we shall describe an analogous set of internal rays in the parameter planes for these maps.

It is a pleasure to acknowledge the fundamental work of Michał Misiurewicz in dynamical systems over the years. Indeed, it was his pathbreaking paper on the dynamics of the complex exponential [10] that originally got me interested in holomorphic dynamics.

\section{Preliminaries. Let}

$$
F_{\lambda}(z)=z^{n}+\lambda / z^{n}
$$

where $n>2$ and $\lambda \in \mathbb{C}$. The Julia set of $F_{\lambda}$, denoted by $J\left(F_{\lambda}\right)$, is defined to be the set of points at which the family of iterates of $F_{\lambda}$ fails to be a normal family in the sense of Montel. There are many other equivalent definitions of the Julia set. For example, the Julia set is the closure of the set of repelling periodic points of $F_{\lambda}$, and, in our special case, it is also the boundary of the set of points whose orbits escape to $\infty$. As a consequence, the Julia set is the set of points on which $F_{\lambda}$ behaves chaotically, since arbitrarily close to any point in $J\left(F_{\lambda}\right)$ there is both a repelling periodic point and a point whose orbit escapes to $\infty$. The complement of the Julia set is called the Fatou set.

One checks easily that $F_{\lambda}$ has $2 n$ free critical points given by $c_{\lambda}=\lambda^{1 / 2 n}$. The origin and $\infty$ are also critical points but they are not free since $\infty$ is fixed and 0 is mapped directly to $\infty$. Despite the large number of free 
critical points for these maps, there are only two critical values: $n$ of the critical points are mapped to $2 \sqrt{\lambda}$ and the other $n$ are mapped to $-2 \sqrt{\lambda}$. In fact, there is really only one critical orbit, since, when $n$ is even, both of the critical values are then mapped to the same point, while, when $n$ is odd, the entire orbits of the critical values are symmetric under $z \mapsto-z$. Thus this family of maps, like the quadratic polynomial family, is a natural one-parameter family of maps. The parameter plane (the $\lambda$-plane) is then a record of the behavior of the free critical orbit, just as in the case of the Mandelbrot set. There are also $2 n$ prepoles at the points $p_{\lambda}=(-\lambda)^{1 / 2 n}$, so $F_{\lambda}\left(p_{\lambda}\right)=0$.

Let $C_{\lambda}$ be the circle given by $|z|=|\lambda|^{1 / 2 n}$. Note that $C_{\lambda}$ contains all of the critical points and prepoles of $F_{\lambda}$. A straightforward computation shows that $F_{\lambda}$ maps $C_{\lambda} 2 n$-to-one onto the straight line connecting the two critical values. We call $C_{\lambda}$ the critical circle and its image the critical line. One may also check that any other circle centered at the origin is mapped $n$-to-one onto an ellipse whose foci are $\pm v_{\lambda}$.

There are several symmetries for these maps. Let $\nu$ be the primitive $2 n$th root of unity. Then we have $F_{\lambda}(\nu z)=\nu^{n} F_{\lambda}(z)$, so it follows easily that $J\left(F_{\lambda}\right)$ is invariant under $z \mapsto \nu z$. Also, let $H_{\lambda}(z)$ be one of the $n$ involutions given by $H_{\lambda}(z)=\lambda^{1 / n} / z$. Then we have $F_{\lambda}\left(H_{\lambda}(z)\right)=F_{\lambda}(z)$, so the Julia set is also preserved by each of these involutions.

The parameter plane for $F_{\lambda}$ also possesses several symmetries. First of all, we have $\overline{F_{\lambda}(z)}=F_{\bar{\lambda}}(\bar{z})$ so that $F_{\lambda}$ and $F_{\bar{\lambda}}$ are conjugate via the map $z \mapsto \bar{z}$. The parameter plane is also symmetric under $z \mapsto \omega z$ where $\omega$ is an $(n-1)$ st root of unity, though the symmetrically located maps are not always conjugate (though their second iterates are conjugate). See [6] for details.

When $|z|$ is large, the term $\lambda / z^{n}$ in the formula for $F_{\lambda}$ is negligible, so $F_{\lambda}(z) \approx z^{n}$ near $\infty$. Consequently, the point at $\infty$ is a superattracting fixed point for $F_{\lambda}$, so we have an immediate basin of attraction $B_{\lambda}$ at $\infty$. Since $F_{\lambda}$ has a pole of order $n$ at 0 , there is an open neighborhood of 0 that is mapped $n$-to-one onto a neighborhood of $\infty$ in $B_{\lambda}$. If the immediate basin of $\infty$ is disjoint from this neighborhood around the origin, then there is an open set about 0 that is mapped $n$-to-one onto $B_{\lambda}$. This set is called the trap door, since any orbit that eventually enters $B_{\lambda}$ must do so by passing through the trap door. We denote the trap door by $T_{\lambda}$.

As in the case of the quadratic polynomials $z^{2}+c$, the orbits of the free critical points may tend to $\infty$. However, unlike the quadratic case, there are three distinct ways these critical orbits escape, and these lead to three different types of Julia sets for these maps. The following theorem was proved in $[6]$. 
Theorem (The escape trichotomy).

1. If $v_{\lambda}$ lies in $B_{\lambda}$, then $J\left(F_{\lambda}\right)$ is a Cantor set.

2. If $v_{\lambda}$ lies in $T_{\lambda} \neq B_{\lambda}$, then $J\left(F_{\lambda}\right)$ is a Cantor set of concentric simple closed curves surrounding the origin.

3. In all other cases, $J\left(F_{\lambda}\right)$ is a connected set. In particular, if $F_{\lambda}^{j}\left(v_{\lambda}\right) \in$ $T_{\lambda} \neq B_{\lambda}$ for some $j \geq 1$, then $J\left(F_{\lambda}\right)$ is a Sierpinski curve.

We remark that case 2 of this theorem does not occur when $n=2$; this is one of the reasons why we restrict $n$ to be larger than 2 . See Figure 1 for a picture of the parameter planes for the cases $n=3$ and $n=4$. In each case, the exterior region contains the parameters for which the Julia set is a Cantor set; this is the Cantor set locus. The small, central disk is the region where the Julia set is a Cantor set of circles; this is the McMullen domain as it was McMullen who first discovered this type of Julia set (see [8]). The complement of these two regions is the connectedness locus. The "holes" in this region are the sets where the Julia set is a Sierpiński curve; we call these regions Sierpiński holes. See [1], [2], and [5] for other ways that Sierpiński curve Julia sets arise in this family.

Note that there are two large Mandelbrot sets along the real axis in the parameter plane for $n=3$ and three when $n=4$. In general, there are $n-1$ symmetrically located Mandelbrot sets in the parameter plane for $z^{n}+\lambda / z^{n}$. These are the so-called principal Mandelbrot sets; their existence was shown in [3]. In this paper we shall prove the existence of infinitely many other Mandelbrot sets some of which are visible in Figure 1.
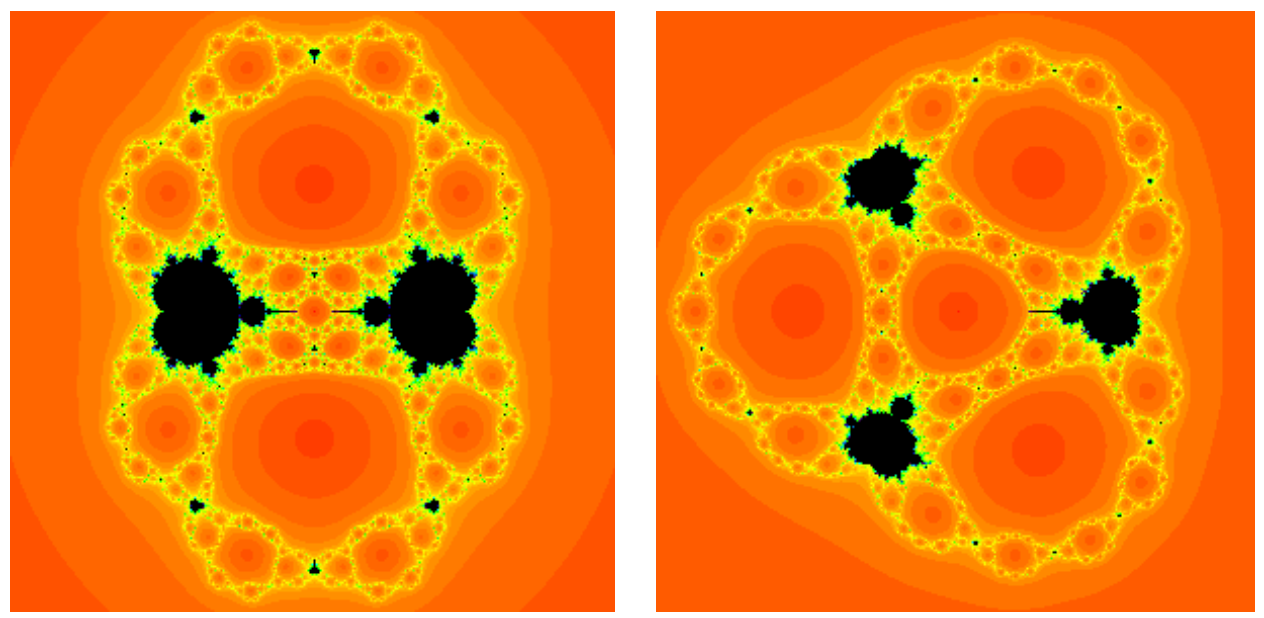

Fig. 1. The parameter planes when $n=3$ and $n=4$

Finally, since $\infty$ is superattracting, there is a Böttcher coordinate in a neighborhood of $\infty$. This is an analytic map $\phi_{\lambda}$ that conjugates $F_{\lambda}$ to $z \mapsto z^{n}$ 
near $\infty$. It is known that

$$
\phi_{\lambda}(z)=\lim _{k \rightarrow \infty}\left(F_{\lambda}^{k}(z)\right)^{n^{-k}} .
$$

Furthermore, $\phi_{\lambda}$ is unique up to multiplication by an $(n-1)$ st root of unity and we may choose $\phi_{\lambda}^{\prime}(\infty)=1$. See [9], [11].

2. Internal rays in the dynamical plane. In this section, for simplicity, we restrict attention to the family of maps

$$
F_{\lambda}(z)=z^{3}+\lambda / z^{3} \text {. }
$$

At the end of this paper we sketch the straightforward modifications needed to extend these results to the case where $n>3$. Because of the $(n-1)$-fold symmetry in the parameter plane noted earlier, we may restrict attention to parameters $\lambda$ that lie in the upper half-plane which we denote by $\mathcal{H}$. Since $F_{\lambda}$ and $F_{-\lambda}$ are conjugate by the map $z \mapsto-z$, the construction below works equally well for parameters for which $\pi<\operatorname{Arg} \lambda<2 \pi$. The case where $\lambda \in \mathbb{R}$ is somewhat special but straightforward; this case will be discussed later.

Let $c_{0}=c_{0}(\lambda)=\lambda^{1 / 6}$ denote the critical point that lies on the real axis when $\lambda \in \mathbb{R}^{+}$and that varies analytically as $\lambda$ ranges through $\mathcal{H}$. Let $c_{j}=c_{j}(\lambda)=c_{0} \exp (2 \pi i j / 6)$, so the $c_{j}$ are arranged in counterclockwise order around the origin as $j$ increases. Three of the critical points (namely, $c_{0}, c_{2}$, and $c_{-2}$ ) are mapped to $v_{\lambda}=2 \sqrt{\lambda}$ which lies in the region $0<\operatorname{Arg} v_{\lambda}<\pi / 2$ the other three critical points are mapped to $-v_{\lambda}$, which lies in the negative of this region.

The straight line connecting the origin to $\infty$ and passing through $c_{j}$ is called a critical point ray and is denoted by $\zeta_{j}$. For $j=0,1,2,3$, let $S_{j}$ be the closed sector bounded by the critcal point rays $\zeta_{j}$ and $\zeta_{j+1}$. Define $S_{-1}$ to be $-S_{1}$ and $S_{-2}$ to be $-S_{2}$. So these sectors are arranged in counterclockwise order about the origin as $S_{0}, S_{1}, S_{2}, S_{3}, S_{-1}, S_{-2}$. The reason for this somewhat peculiar ordering will become clear when we define the internal rays for these maps. $S_{j}$ is called a prepole sector since there is a unique prepole at the "center" of each $S_{j}$. One checks easily that the image of $\zeta_{j}$ under $F_{\lambda}$ is a straight ray connecting one of the two critical values to $\infty$; we call this ray a critical value ray. Note that, since $\lambda$ does not lie in $\mathbb{R}^{+}$, the image of each critical point ray lies in the interior of either $S_{0}$ or $S_{3}$ for each $\lambda$. As a consequence, it follows that $F_{\lambda}$ maps each of $S_{1}, S_{2}, S_{-1}$, and $S_{-2}$ univalently over a region that contains the union of these four sectors. The internal rays that we consider in this paper will always lie in one of these four sectors.

Let $\gamma_{\lambda}$ denote the circle of radius $r=r(\lambda)$ centered at the origin and lying in $B_{\lambda}$. We choose $r$ so that $r(\lambda)$ depends smoothly on $\lambda$ and $F_{\lambda}\left(\gamma_{\lambda}\right)$ 


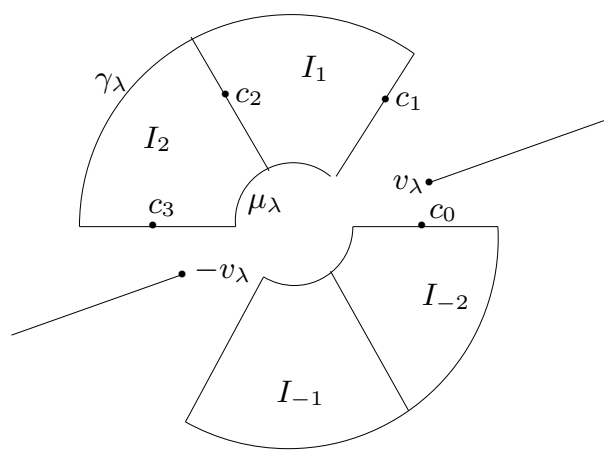

Fig. 2. The region $\mathcal{I}$

lies strictly outside $\gamma_{\lambda}$. Let $\mu_{\lambda}$ be the circle of radius $|\lambda|^{1 / 3} / r$; that is, $\mu_{\lambda}=$ $H_{\lambda}\left(\gamma_{\lambda}\right)$. So $F_{\lambda}\left(\gamma_{\lambda}\right)=F_{\lambda}\left(\mu_{\lambda}\right)$ and $F_{\lambda}$ also maps $\mu_{\lambda}$ strictly outside $\gamma_{\lambda}$.

Now consider the closed region in each $S_{j}$ bounded on the outside by $\gamma_{\lambda}$ and on the inside by $\mu_{\lambda}$. Denote this region by $I_{j}=I_{j}(\lambda)$. Let $\mathcal{I}=\mathcal{I}(\lambda)$ denote the union of the four regions $I_{1}, I_{2}, I_{-1}$, and $I_{-2}$. The two regions $I_{0}$ and $I_{3}$ are not contained in $\mathcal{I}$. See Figure 2 . Let $\Lambda_{\lambda}$ denote the set of points whose orbits remain in $\mathcal{I}$ for all iterations. Then we have:

Proposition. The set $\Lambda_{\lambda}$ is homeomorphic to a Cantor set for each $\lambda \in \mathcal{H}$. Also, $F_{\lambda} \mid \Lambda_{\lambda}$ is conjugate to the one-sided shift on the four symbols $\pm 1, \pm 2$. The sets $\Lambda_{\lambda}$ vary analytically with $\lambda \in \mathcal{H}$.

Proof. For each $\lambda \in \mathcal{H}, F_{\lambda}$ maps the boundary curves $\gamma_{\lambda}$ and $\mu_{\lambda}$ strictly outside $\gamma_{\lambda}$, and hence outside $\mathcal{I}$. Also, $F_{\lambda}$ maps the two critical point ray boundaries of each of the $I_{j}$ in $\mathcal{I}$ to the two critical value rays, both of which lie in the interior of $S_{0}$ or $S_{3}$ for each $\lambda \in \mathcal{H}$ and hence also outside $\mathcal{I}$. Therefore it follows that $F_{\lambda}$ maps each $I_{j}$ in $\mathcal{I}$ univalently onto a region that completely covers each of the other $I_{k}$ in $\mathcal{I}$. Standard arguments from complex dynamics then give that $\Lambda_{\lambda}$ is a Cantor set with $F_{\lambda} \mid \Lambda_{\lambda}$ conjugate to the one-sided shift map on the four symbols $\pm 1, \pm 2$. Since the $I_{j}$ vary analytically with $\lambda$, we have that the points in $\Lambda_{\lambda}$ also vary analytically with $\lambda$.

REMARK. We emphasize that the set $\Lambda_{\lambda}$ is only a subset of $J\left(F_{\lambda}\right)$; indeed, $J$ itself may be connected.

Let $\Sigma$ denote the space of one-sided sequences of the four symbols $\pm 1, \pm 2$, and let $\sigma: \Sigma \rightarrow \Sigma$ be the one-sided shift map. By the preceding proposition, each point in $\Lambda_{\lambda}$ corresponds a unique itinerary $s=\left(s_{0}, s_{1}, s_{2}, \ldots\right) \in \Sigma$.

We shall next be concerned with a special subset $\Gamma$ of $\Sigma$. The subset $\Gamma$ consists of all sequences in $\Sigma$ satisfying:

1. the digits -1 and 2 can only be followed by either 1 or 2 ;

2. the digits 1 and -2 can only be followed by either -1 or -2 . 
Clearly, $\Gamma$ is a shift-invariant subset of $\Sigma$. Indeed, the shift map on $\Gamma$ can be thought of as the subshift of finite type on the four symbols $\pm 1, \pm 2$ that obeys the above rules.

We can think of $\Gamma$ in another way. Let $\tau$ denote the angle-tripling map on the unit circle, i.e., $\tau(\theta)=3 \theta \bmod 1$ where $\theta$ is defined mod 1 . Consider the four arcs in the circle given by $J_{1}=[1 / 4,1 / 3], J_{2}=[5 / 12,1 / 2], J_{-1}=$ $[3 / 4,5 / 6]$, and $J_{-2}=[11 / 12,1]$. Then it follows immediately that $\tau$ expands $J_{1}$ and $J_{-2}$ over both $J_{-1}$ and $J_{-2}$, while $\tau$ expands $J_{2}$ and $J_{-1}$ over $J_{1}$ and $J_{2}$. Thus the set of points on the unit circle whose orbits under $\tau$ remain for all iterations in these four arcs is homeomorphic to the set $\Gamma$ and, moreover, the map $\tau$ on this set is conjugate to the shift map on $\Gamma$. With a slight abuse of notation, we denote the set of all of these angles on the unit circle by $\Gamma$ as well. We similarly denote the itinerary of the angle $\theta$ under $\tau$ by $s(\theta)=\left(s_{0}, s_{1}, s_{2}, \ldots\right) \in \Gamma$.

As a remark, we could equally well have defined $\Gamma$ to be the set of angles that remain in the two arcs $[1 / 4,1 / 2]$ and $[3 / 4,1]$ under angle tripling mod 1. We prefer the above definition since it matches with the subshift definition given earlier.

As mentioned earlier, since $\infty$ is superattracting, we have a Böttcher coordinate $\phi_{\lambda}: B_{\lambda} \rightarrow \mathbb{C}-\overline{\mathbb{D}}$. This is an analytic map that conjugates $F_{\lambda}$ in a neighborhood of $\infty$ to the map $z \mapsto z^{3}$ also near $\infty$. We may choose $\phi_{\lambda}$ so that $\phi_{\lambda}^{\prime}(\infty)=1$. It is known that, when $\lambda$ lies in the connectedness locus or in the McMullen domain, $\phi_{\lambda}$ may be extended to a map that takes $B_{\lambda}$ univalently onto the exterior of the closed unit disk in the Riemann sphere (see [9], [11]). When $\lambda$ lies in the Cantor set locus, the domain of $\phi_{\lambda}$ is smaller due to the presence of critical points in $B_{\lambda}$.

Given an angle $\theta \bmod 1$, the external ray of angle $\theta$, denoted by $\xi_{\lambda}^{\theta}(t)$, is the image of the straight ray $t \mapsto t \exp (2 \pi i \theta), t \in(1, \infty]$, under the inverse map $\phi_{\lambda}^{-1}$. We set $\xi_{\lambda}^{\theta}(\infty)=\infty$ for each $\theta \in \Gamma$. When $\lambda$ lies in the connectedness locus or the McMullen domain, each $\xi_{\lambda}^{\theta}(t)$ is defined for all $t>1$, and all of these external rays accumulate on the boundary of $B_{\lambda}$, though they may not limit on a unique landing point in $\partial B_{\lambda}$ if $\partial B_{\lambda}$ is not locally connected. However, the following proposition shows that we always do have a unique landing point whenever the angle $\theta$ lies in $\Gamma$; in particular, this also occurs in case $\lambda$ lies in the Cantor set locus.

Proposition. Let $\theta \in \Gamma$ and $\lambda \in \mathcal{H}$. Then the external ray $\xi_{\lambda}^{\theta}$ may be extended so that it lands on (i.e., tends to a limit as $t \rightarrow 1$ at) the unique point in $\Lambda_{\lambda}$ whose itinerary is the same as that of $\theta$ under the angle-tripling $\operatorname{map} \tau$.

Proof. Recall that each of the prepole sectors $S_{1}, S_{2}, S_{-1}$, and $S_{-2}$ is mapped univalently over the union of all four of these sectors by $F_{\lambda}$ and 
that the exterior of the circle $\gamma_{\lambda}$ of radius $r=r(\lambda)$ lies in $B_{\lambda}$. Given $\lambda \in \mathcal{H}$, let $N_{r}=\{z|| z \mid \geq r(\lambda)\}$ and let $N_{r}^{j}=N_{r} \cap S_{j}$. Since $\phi_{\lambda}^{\prime}(\infty)=1$, we may further assume that $r(\lambda)$ is chosen large enough so that, for each $\theta \in \Gamma$, the portion of the external ray $\xi_{\lambda}^{\theta}$ lying in $N_{r}$ is precisely the set of points in $N_{r}$ whose orbits have the exact same itinerary through the sets $N_{r}^{j}$ as the angle $\theta$ has under $\tau$, namely $s(\theta)$.

Now we can extend this concept to the entire collection of sectors $S_{j}$ with $j= \pm 1, \pm 2$ by pulling back the portions of the external rays that lie in $N_{r}$ by $F_{\lambda}^{-1}$. More precisely, take the portion of the external ray with itinerary $\left(s_{1}, s_{2}, s_{3}, \ldots\right)$ that lies in $N_{r}^{s_{1}}$ and pull it back under $F_{\lambda}^{-1}$ into $S_{s_{0}}$. This extends the portion of $\xi_{\lambda}^{\theta}$ lying in $N_{r}^{s_{0}}$ further back into $S_{s_{0}}$. Continuing in this fashion with the portion of the ray with itinerary $\left(s_{n}, s_{n+1}, s_{n+2}, \ldots\right)$ in $N_{r}^{s_{n}}$ shows that the entire external ray $\xi_{\lambda}^{\theta}(t)$ for all $t>1$ is defined and lies in $S_{s_{0}}$.

Now suppose that $\xi_{\lambda}^{\theta}(t)$ accumulates on some point $z \in \mathbb{C}$ as $t \rightarrow 1$. Then $z$ cannot lie in $B_{\lambda}$. This follows immediately when $\lambda$ does not lie in the Cantor set locus since $\phi_{\lambda}$ is defined and univalent on all of $B_{\lambda}$. If $\lambda$ does lie in the Cantor set locus and $z$ lies in $B_{\lambda}$, then we may iterate $F_{\lambda}$ forward enough times so that $F_{\lambda}^{k}(z)$ lies in the region where the Böttcher coordinate is defined and analytic. But then the corresponding external ray would accumulate on a point in $B_{\lambda}$ as $t \rightarrow \infty$ and that cannot happen. Thus $\xi_{\lambda}^{\theta}(t)$ must accumulate on a point in $J\left(F_{\lambda}\right)$. But this accumulation point must then have the same itinerary through the $S_{j}$ as that of $\theta$. However, we know that there is a unique such point in the Julia set, namely the point in $\Lambda_{\lambda}$ with this itinerary. Thus each external ray $\xi_{\lambda}^{\theta}$ lands at the unique point in $\partial B_{\lambda}$ whose itinerary is the same as that of $\theta$ whenever $\theta \in \Gamma$.

Given an itinerary $s=s(\theta)=\left(s_{0}, s_{1}, s_{2}, \ldots\right)$, we define the negative of $s$ to be the itinerary $-s(\theta)=\left(-s_{0},-s_{1},-s_{2}, \ldots\right)$ and the shift of $s$ to be $\sigma(s)=\left(s_{1}, s_{2}, s_{3}, \ldots\right)$. Also, let $\hat{\theta}=\theta+1 / 2 \bmod 1$ and $\hat{\tau}(\theta)=\tau(\theta)+1 / 2$ $\bmod 1$. Consequently, $s(\hat{\tau}(\theta))=-\sigma(s(\theta))$. For example, if $\theta=1 / 3$, then $s(1 / 3)=(1, \overline{-2})$ and $\hat{\tau}(1 / 3)=1 / 2$. So we have $s(\hat{\tau}(1 / 3))=s(1 / 2)=(\overline{2})=$ $-\sigma(s(1 / 3))$ as required.

Let $\mathbb{S}$ denote the union of the four sectors $S_{ \pm 1}$ and $S_{ \pm 2}$. Given a point $z$ whose entire orbit lies in $\mathbb{S}$, we may define its itinerary as above to be $s(z)=\left(s_{0}, s_{1}, s_{2}, \ldots\right)$ where $s_{j}=k$ implies that $F_{\lambda}^{j}(z) \in S_{k}$. Note that, even though a pair of sectors overlap along the critical point rays, points on these rays are mapped immediately outside of $\mathbb{S}$. Consequently, the itinerary $s(z)$ is always well-defined when the entire orbit of $z$ lies in $\mathbb{S}$.

Definition. Let $\theta \in \Gamma$ and suppose that $s(\theta)=\left(s_{0}, s_{1}, s_{2}, \ldots\right)$. The full ray of angle $\theta$, denoted by $\omega_{\lambda}^{\theta}$, consists of all points in $\mathbb{S}$ whose itinerary is of the form $\left(s_{0}, \pm s_{1}, \pm s_{2}, \pm s_{3}, \ldots\right)$ together with the origin and $\infty$. That is, $\omega_{\lambda}^{\theta}$ 
consists of all points in $S_{s_{0}}$ whose itinerary is the same as that of $\theta$, except that we may change the sign of any of the $s_{j}$ with $j>0$.

Note that in this definition we always choose $\theta$ so that $s(\theta) \in \Gamma$. However, the points on the full ray with angle $\theta$ have itineraries that lie in the larger set $\Sigma$, not just $\Gamma$. Also, note that the external ray $\xi_{\lambda}^{\theta}$ is part of the full ray $\omega_{\lambda}^{\theta}$ since all points on the external ray have itinerary $s(\theta)$. This implies that the set of points in $\Lambda_{\lambda}$ whose itinerary corresponds to an angle in $\Gamma$ lies in $\partial B_{\lambda}$ when $\lambda$ lies in the connectedness locus or the McMullen domain. This is the dynamical significance of the set $\Gamma$. We also denote the pair of full rays $\omega_{\lambda}^{\theta} \cup \omega_{\lambda}^{\hat{\theta}}$ by $\Omega_{\lambda}^{\theta}$ (or, equally well, $\Omega_{\lambda}^{\hat{\theta}}$ ). Finally, this defintion explains why we choose the peculiar ordering of the $S_{j}$ when $j$ is negative; we only have to change the signs of the $s_{j}$ to determine all points in the full ray.

Definition. The internal ray of angle $\theta$, denoted $\nu_{\lambda}^{\theta}$, is the complement of the external ray $\xi_{\lambda}^{\theta}$ in the full ray $\omega_{\lambda}^{\theta}$.

TheOrem. Let $\lambda \in \mathcal{H}$ and $\theta \in \Gamma$. Then the full ray $\omega_{\lambda}^{\theta}$ has the following properties:

1. $\omega_{\lambda}^{\theta}$ meets $J\left(F_{\lambda}\right)$ in a Cantor set of points.

2. $\omega_{\lambda}^{\theta}$ is mapped univalently onto the pair of full rays $\Omega_{\lambda}^{\tau(\theta)}$.

3. $\omega_{\lambda}^{\theta}$ is a continuous curve extending from 0 to $\infty$, i.e., there is a continuous, one-to-one map taking the closed half-line $[0, \infty]$ onto $\omega_{\lambda}^{\theta}$.

Proof. For part 1, consider the portion of $\omega_{\lambda}^{\theta}$ that lies inside the bounded region $I_{s_{0}} \subset S_{s_{0}}$ and contains no points whose orbits tend to $\infty$, i.e., the entire orbit lies in $\mathcal{I}$. This portion of $\omega_{\lambda}^{\theta}$ corresponds exactly to the set of points in $\Lambda_{\lambda}$ whose itinerary in $\Sigma$ is a sequence of the form $\left(s_{0}, \pm s_{1}, \pm s_{2}, \pm s_{3}, \ldots\right)$. As shown earlier, there is exactly one such point for each given sequence, and these points lie in $J\left(F_{\lambda}\right)$. It follows immediately that the points with these itineraries form a Cantor set in $I_{s_{0}}$.

For part 2, we see that $\omega_{\lambda}^{\theta}$ can be divided into two disjoint subsets, those points with itinerary $\left(s_{0},+s_{1}, \pm s_{2}, \pm s_{3}, \pm s_{4}, \ldots\right)$ and those with itinerary $\left(s_{0},-s_{1}, \pm s_{2}, \pm s_{3}, \pm s_{4}, \ldots\right)$. These portions of $\omega_{\lambda}^{\theta}$ are mapped one-to-one by $F_{\lambda}$ onto $\omega_{\lambda}^{\tau(\theta)}$ and $\omega_{\lambda}^{\hat{\tau}(\theta)}$ respectively since $F_{\lambda} \mid S_{s_{0}}$ is univalent.

Finally, for part 3 , we first note that there is a preimage of the external ray $\xi_{\lambda}^{\hat{\tau}(\theta)}$ that lies in $S_{s_{0}}$ and connects a point in $J\left(F_{\lambda}\right)$ to the origin. This arc then lies in $\omega_{\lambda}^{\theta}$ since its itinerary is $\left(s_{0},-s_{1},-s_{2},-s_{3} \ldots\right)$. If $\lambda$ lies in the connectedness locus or the McMullen domain, then this arc lies in the trap door.

Now consider the pair of full rays $\omega_{\lambda}^{\theta}$ and $\omega_{\lambda}^{\hat{\theta}}$. We first claim that this set of points is a closed, connected set. To see this, note that the set of points whose itinerary begins with either $s_{0}$ or $-s_{0}$ is a closed connected set in $\overline{\mathbb{C}}$ 
(here as elsewhere we include both the origin and $\infty$ in this set). Indeed, this set is just the two closed sectors $S_{s_{0}}$ and $S_{-s_{0}}$. In the Riemann sphere, this set is a pair of closed "disks" that meet at two points, namely at 0 and $\infty$. Recall that each of these sectors is mapped univalently over $S_{s_{1}} \cup S_{-s_{1}}$. We therefore have a preimage of $S_{s_{1}} \cup S_{-s_{1}}$ in each of $S_{s_{0}}$ and $S_{-s_{0}}$, and each of these preimages connects 0 to $\infty$. So the set of points whose itinerary begins $\pm s_{0}, \pm s_{1}$ is then a string of four closed disks that are contained in $S_{s_{0}} \cup S_{-s_{0}}$. Each of these disks meet exactly two of the other disks, one at either 0 or $\infty$ and the other at one of the two preimages of 0 that lie in the previous set. Then the preimage of this set is a string of eight closed disks, each of which meets exactly two others at $0, \infty$, or their first or second preimages, and this string of disks is again contained in the previous set. Continuing inductively, we see that the set of points with this set of itineraries is a nested intersection of closed, connected sets in $\overline{\mathbb{C}}$, each of which is a string of $2^{n}$ closed disks, and each of these disks meets exactly two others at different points on its boundary. Hence the intersection is also a closed, connected set.

Now any point in $\omega_{\lambda}^{\theta}$ that is not in $J\left(F_{\lambda}\right)$ must lie in the Fatou set. If such a point does not eventually tend to $\infty$, its orbit must be bounded. But then this point would correspond to some point in $\Lambda_{\lambda}$ which then must lie in $J\left(F_{\lambda}\right)$. Therefore, the orbits of all other points in $\omega_{\lambda}^{\theta}$ must eventually tend to $\infty$. This implies that they must, at some iteration, say the $n$ th, land on an external ray in $\mathbb{S}$ whose itinerary is of the form $\left( \pm s_{n}, \pm s_{n+1}, \pm s_{n+2}, \ldots\right)$. But there are only two choices for such an external ray. To see this, recall that the itinerary of any external ray whose orbit lies in $\mathbb{S}$ corresponds to that of an angle in $\Gamma$. We know two possible such external angles, namely $\tau^{n}(\theta)$ and $\hat{\tau}^{n}(\theta)$, i.e., the itineraries $\left(s_{n}, s_{n+1}, s_{n+2}, \ldots\right)$ and $\left(-s_{n},-s_{n+1},-s_{n+2}, \ldots\right)$. Any other possible itinerary involving these digits would then change some digit following $s_{n}$ or $-s_{n}$ in this sequence. Say $s_{n+k}$ is changed where $k>0$. Then, to keep this sequence in $\omega_{\lambda}^{\theta}$, we can only change $s_{n+k}$ to $-s_{n+k}$. But, if $s_{n+k}$ is allowed to follow $s_{n+k-1}$, then the rules governing the itineraries in $\Gamma$ say that $-s_{n+k}$ cannot follow $s_{n+k-1}$.

As a consequence, $\omega_{\lambda}^{\theta}$ consists of the external ray $\xi_{\lambda}^{\theta}$, the preimage of $\xi_{\lambda}^{\hat{\tau}(\theta)}$ connecting to the origin, the Cantor set of points described earlier, and all points with the required itinerary that eventually land on the external rays with angle $\tau^{n}(\theta)$ or $\hat{\tau}^{n}(\theta), n>1$, together with 0 and $\infty$. But, for each $n>1$, there are only a finite number of such preimages, in fact, exactly $2^{n-1}$ such arcs. Each of these curves then connects to a single point in the Cantor set portion of $\omega_{\lambda}^{\theta}$ since we know that the original external rays of angle $\pm \theta$ have this property. The other endpoint of these curves is one of the $2^{n-2} n$th preimages of $\infty$ in $\omega_{\lambda}^{\theta}$. Since, as we showed above, the union of all of the points in $\omega_{\lambda}^{\theta}$ is closed and connected, it then follows that the entire 
set must be a closed curve that extends from 0 to $\infty$. Here the preimages of the two external rays with angle $\pm \tau^{n}(\theta)$ fill in the "gaps" in the Cantor set, since there are a pair of preimages of the external rays that connect to each preimage of 0 and also to a pair of points in $J\left(F_{\lambda}\right)$. This completes the proof.

As a consequence of this result, we can identify any point on the internal ray $\nu_{\lambda}^{\theta}$ uniquely as follows.

Theorem (Internal ray specification). Let $z \in \nu_{\lambda}^{\theta}$. Then we may identify z uniquely by:

1. If $z \in \nu_{\lambda}^{\theta} \cap \Lambda_{\lambda}$, then we simply specify its itinerary in $\Sigma$.

2. If $z$ lies in the complement of this set in $\nu_{\lambda}^{\theta}$, then there is a first iterate, say the $k$ th where $k \geq 1$, at which the orbit of this point reaches $B_{\lambda}$. Then this point lies on the external ray with angle either $\tau^{k}(\theta)$ or $\hat{\tau}^{k}(\theta)$, so on some point of the form either $\xi_{\lambda}^{\tau^{k}(\theta)}(t)$ or $\xi_{\lambda}^{\hat{\tau}^{k}(\theta)}(t)$. So we may specify this point uniquely by giving $\theta$, $t$, and which of the two external angles $\tau^{k}(\theta)$ or $\hat{\tau}^{k}(\theta)$ the orbit of $z$ lands on.

REMARK. As mentioned above, the case where $\lambda$ lies in the lower halfplane is entirely analogous, as the parameter plane is symmetric with respect to complex conjugation. The case where $\lambda \in \mathbb{R}$ is somewhat different. For example, if $\lambda \in \mathbb{R}^{+}$, the critical values have orbits that remain in $\mathbb{R}$. If this orbit is bounded, we can then extend the definition of the internal rays to include those in the sectors $S_{0}$ and $S_{3}$. The difference arises in that certain external rays now separate into a pair of internal rays when they enter the Julia set. For example, the external ray along $\mathbb{R}^{+}$now joins up with the internal ray with itinerary $\overline{0}$ in $S_{0}$ and also with the internal ray $\overline{2}$ in $S_{2}$. Despite these differences, most of the above construction then proceeds in similar fashion, though the symbolic dynamics is now more complicated. We leave the details of this special case to the reader.

3. Intertwined internal rays. In this section, we show how the internal rays whose angle $\theta$ lies in $\Gamma$ are always "intertwined." By intertwined, we mean that each of these internal rays crosses infinitely many other such internal rays. More precisely, we shall prove:

Theorem (Intertwined internal rays). Let $\theta \in \Gamma$ and suppose the first digit of $s(\theta)$ is $s_{0}$. Then the internal ray of angle $\theta$ crosses every other internal ray whose angle lies in $\Gamma$ and whose itinerary begins with $s_{0}$ at some interior point of $S_{s_{0}}$.

Proof. For definiteness, we first consider the external rays that lie in the sector $S_{2}$. These external rays are mapped univalently onto the set of all external rays of angle $\tau(\theta) \in \Gamma$ lying in $S_{1} \cup S_{2}$. Moreover, the external rays 
in $S_{2}$ are arranged in the exact same order in $S_{2}$ as $\theta$ is ordered in $\Gamma$. That is, if $\theta_{1}<\theta_{2}$, then $\xi_{\lambda}^{\theta_{1}}$ lies above $\xi_{\lambda}^{\theta_{2}}$ in the sector $S_{2}$.

Now each external ray $\xi_{\lambda}^{\theta}$ in $S_{2}$ connects to a unique internal ray $\nu_{\lambda}^{\theta}$ that also lies in $S_{2}$, and each of these internal rays contains an arc in the Fatou set that extends from the origin to a first point in $J\left(F_{\lambda}\right)$. Call this $\operatorname{arc} \mu_{\lambda}^{\theta}$. Then $\mu_{\lambda}^{\theta}$ is the preimage in $S_{2}$ of the external ray $\xi_{\lambda}^{\hat{\tau}(\theta)}$. The collection of $\operatorname{arcs} \mu_{\lambda}^{\theta}$ in $S_{2}$ is just the collection of all preimages of external rays that lie in $S_{-1} \cup S_{-2}$ (with angles in $\Gamma$ ).

We claim that the arcs $\mu_{\lambda}^{\theta}$ are arranged around the origin in $S_{0}$ in the exact opposite order of the $\xi_{\lambda}^{\theta}$ as $\theta$ increases. This occurs since, close to the origin, $F_{\lambda} \approx \lambda / z^{3}$, so $F_{\lambda}$ reverses the ordering of rays near the origin as they are mapped to the external rays near $\infty$. That is, if $\theta_{1}<\theta_{2}$, then $\mu_{\lambda}^{\theta_{2}}$ lies above $\mu_{\lambda}^{\theta_{1}}$ in the natural ordering of these rays around the origin in $S_{2}$. Since $\xi_{\lambda}^{\theta}$ must connect to $\mu_{\lambda}^{\theta}$ for each $\theta$, it then follows that any two internal rays of different angles must cross at some point in $S_{2}$ that is not the origin nor $\infty$.

For rays lying in the other sectors similar arguments work as well.

As a specific example of intertwining, let $\theta=1 / 2$. Then $s(1 / 2)=(\overline{2})$, and $\xi_{\lambda}^{1 / 2}$ is the fixed external ray lying in $S_{2}$. Similarly, when $\theta=0, \xi_{\lambda}^{0}$ lies in $S_{-2}$ and has itinerary $(\overline{-2})$. So $\omega_{\lambda}^{1 / 2}$ is mapped over the pair of full rays $\omega_{\lambda}^{1 / 2}$ and $\omega_{\lambda}^{0}$, which we have named $\Omega_{\lambda}^{1 / 2}$ (or $\Omega_{\lambda}^{0}$ ). Similarly, $\theta=1 / 4$ has itinerary $s(1 / 4)=(\overline{1,-1})$ so $\xi_{\lambda}^{1 / 4}$ lies in $S_{1}$ and is mapped to $\xi_{\lambda}^{3 / 4}$ in $S_{-1}$, while $\xi_{\lambda}^{3 / 4}$ is mapped back to $\xi_{\lambda}^{1 / 4}$. So $\omega_{\lambda}^{1 / 4}$ lies in $S_{1}$ and is mapped over $\Omega_{\lambda}^{1 / 4}$, while $\omega_{\lambda}^{3 / 4}$ lies in $S_{-1}$ and is also mapped onto the same pair of full rays. Note that $\Omega_{\lambda}^{1 / 2}$ is a simple closed curve in the Riemann sphere that passes through the origin and $\infty$ and lies in $S_{2} \cup S_{-2}$, while $\Omega_{\lambda}^{1 / 4}$ is a similar simple closed curve that lies in $S_{1} \cup S_{-1}$. Hence these curves only meet at 0 and $\infty$. Also note that $F_{\lambda}\left(\Omega_{\lambda}^{1 / 2}\right)=\Omega_{\lambda}^{1 / 2}$ and $F_{\lambda}\left(\Omega_{\lambda}^{1 / 4}\right)=\Omega_{\lambda}^{1 / 4}$; indeed, these are the only pairs of full rays that are mapped over themselves by $F_{\lambda}$.

Now consider the preimages of $\Omega_{\lambda}^{1 / 2}$ and $\Omega_{\lambda}^{1 / 4}$ in $\mathbb{S}$. One computes that $F_{\lambda}\left(\Omega_{\lambda}^{1 / 3}\right)=\Omega_{\lambda}^{0}=\Omega_{\lambda}^{1 / 2}$ and $F_{\lambda}\left(\Omega_{\lambda}^{5 / 12}\right)=\Omega_{\lambda}^{1 / 4}$. Each of the two curves $\Omega_{\lambda}^{1 / 3}$ and $\Omega_{\lambda}^{5 / 12}$ must pass through the two preimages of 0 in the pair of sectors in which they reside, so $\Omega_{\lambda}^{5 / 12}$ meets $\Omega_{\lambda}^{1 / 2}$ in a total of four points, the origin, $\infty$, and the two preimages of 0 . Similarly, $\Omega_{\lambda}^{1 / 3}$ meets $\Omega_{\lambda}^{1 / 4}$ in four points. See Figure 3. In similar fashion, one computes that the preimage of $\Omega_{\lambda}^{5 / 12}$ in $S_{2} \cup S_{-2}$, i.e., $\Omega_{\lambda}^{17 / 36}$, meets $\Omega_{\lambda}^{1 / 2}$ in eight points, the previous four points plus four of the second preimages of 0 lying in this region. Similarly, the preimages of $\omega_{\lambda}^{5 / 12}$ in $S_{1} \cup S_{-1}$, namely $\Omega_{\lambda}^{11 / 36}$, meets $\Omega_{\lambda}^{1 / 3}$ in eight points. 


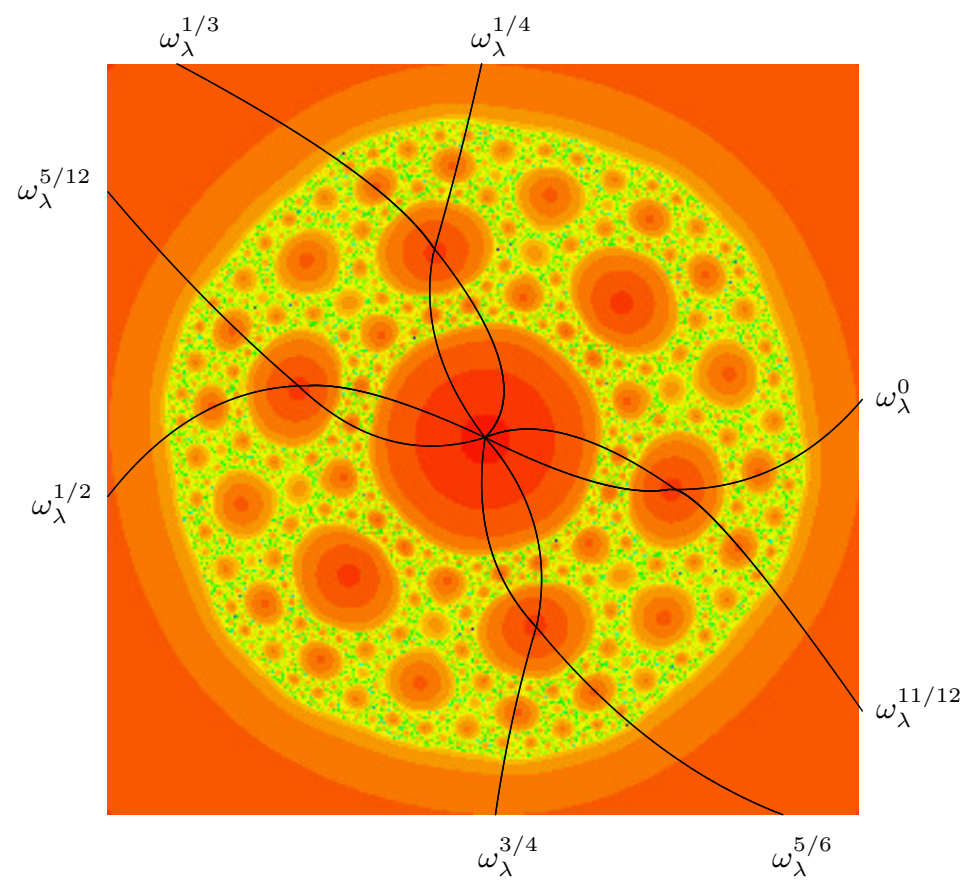

Fig. 3. Some of the rays in the dynamical plane for a fixed $\lambda \in \mathcal{H}$

Continuing in this fashion, we see that any full ray that is a $j$ th preimage of $\Omega_{\lambda}^{1 / 2}$ or $\omega_{\lambda}^{1 / 4}$ meets these curves in exactly $2^{j}$ points.

In particular, note that every internal ray whose itinerary begins with the digit $s_{0}$ must pass through the preimage of 0 that lies in the sector $S_{s_{0}}$. Indeed, infinitely many internal rays whose itinerary is allowable cross at each $k$ th preimage of 0 .

4. A model for the internal rays. In this section we construct a piecewise linear model that exhibits the structure of the internal rays described in the previous two sections. The model for this collection of rays will be drawn in the Sierpinski carpet. This is not intended to say that the Julia sets through which we have drawn the internal rays are always Sierpiński curves; they sometimes are but they need not be such sets. We use the carpet only to facilitate the drawing of the model for these internal rays.

Recall that the Sierpiński carpet consists of infinitely many smaller copies of itself. At stage one, we have the entire carpet. At stage 2, we may break the carpet up into eight copies of itself, each of which is one-third the size of the original. At stage 3 , there are $8^{2}$ copies of the carpet that are $1 / 3^{2}$ the size of the original, and so forth. We will draw three types of internal straight rays in certain of these self-similar portions of the carpet: horizontal rays, 
diagonal rays, and connecting rays. By a horizontal ray, we always mean a straight line passing left to right through the center of a Sierpinski carpet, and by a diagonal ray, we always mean a straight line connecting the upper left corner of a carpet to the lower right corner.

We proceed inductively. At stage one, we draw a horizontal and diagonal ray through the entire carpet. The horizontal ray corresponds to the internal rays $\nu_{\lambda}^{0} \cup \nu_{\lambda}^{1 / 2}$ while the diagonal ray corresponds to $\nu_{\lambda}^{1 / 4} \cup \nu_{\lambda}^{3 / 4}$. Note that these rays meet only at the center of the carpet.

Note that these two rays cross through four of the eight copies of the carpet at stage 2 . In each of these four smaller carpets, we either have a smaller diagonal or a smaller horizontal ray. So, at this stage, we add a new ray to this carpet of the opposite type; that is, if there is a diagonal ray in this stage 2 carpet, we add a horizontal ray. So we have added four portions of rays so far. Now what we do is join up these portions of the rays with connecting rays which are straight lines passing through the center of the original carpet and joining the endpoints of a pair of rays just constructed. That is, we join up the two new horizontal rays with a connecting ray and the two new diagonal rays as well. See Figure 4 . This produces two new rays that correspond to the internal rays $\nu_{\lambda}^{1 / 3}, \nu_{\lambda}^{5 / 12}, \nu_{\lambda}^{5 / 6}$, and $\nu_{\lambda}^{11 / 12}$.

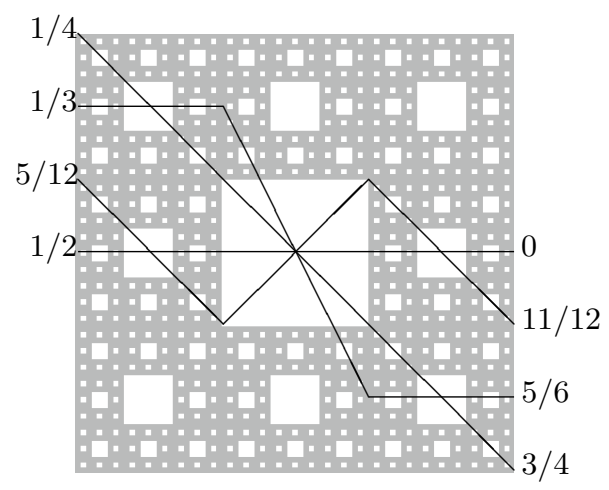

Fig. 4. The first two stages of the construction of the piecewise linear model for the intertwined rays. The rays that correspond to $\nu_{\lambda}^{p / q}$ in Figure 3 are indicated by $p / q$.

Now we proceed inductively. At stage 3, our previously constructed (in stage 1 and stage 2) diagonal and horizontal rays (not the connecting rays) cross through exactly $4^{2}$ copies of the carpet at stage 3 , so we again adjoin a ray of the opposite type in each of these carpets. Then we connect up these rays as follows. First, inside the smaller stage 2 carpets, we pass a connecting line through the center of this smaller carpet and join the ends of symmetrically located rays. Then, in the original carpet, we connect up the endpoints with connecting rays passing through the center of the largest 


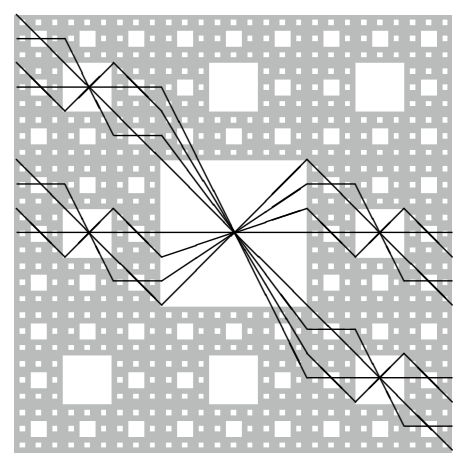

Fig. 5. The first three stages of the construction of the piecewise linear model for the intertwined rays.

carpet. See Figure 5. Taking the limit of this process yields an uncountable collection of curves in the carpet. Given a vertical line through the carpet, this collection of curves meets this line in a Cantor set of points provided that the vertical line does not pass through the center of any of the complementary squares. On the other hand, this collection of curves meets the vertical lines that pass through the centers of the complementary squares in only finitely many points, indeed, in exactly $2^{k-1}$ points if the complementary square is at the center of a stage $k$ carpet.

One checks easily that, using the mapping properties of $F_{\lambda}$ on the various sectors $S_{j}$, this construction corresponds exactly to that of the internal rays in the dynamical plane.

5. Baby Mandelbrot sets. Our goal in this section is to prove the existence of infinitely many disjoint copies of the Mandelbrot set in the parameter plane for $z^{3}+\lambda / z^{3}$. We continue to assume that $\lambda$ lies in $\mathcal{H}$, the upper half-plane, at least initially. Let $\mathcal{R}_{\lambda}$ denote the collection of all full rays whose angle $\theta$ lies in $\Gamma$. The set $\mathcal{R}_{\lambda}$ is a closed subset of the Riemann sphere whose complement consists of a countable collection of open disks (recall that 0 and $\infty$ lie on all of the full rays). One of these complementary disks contains the critical value $v_{\lambda}$ that lies in the upper half-plane; call this disk $U_{0}$. Note that $U_{0}$ contains the entire sector $S_{0}$ as well as portions of both $S_{1}$ and $S_{-2}$. Another complementary disk is $U_{3}=-U_{0}$. This disk contains the entire sector $S_{3}$ and also pieces of $S_{2}$ and $S_{-1}$. Note that $U_{0}$ contains the critical points $c_{0}$ and $c_{1}$ that lie on the boundary of $S_{0}$, while $U_{3}$ contains the critical points $c_{3}$ and $c_{-2}$ lying on the boundary of $S_{3}$. See Figure 6 .

There are two other open sets in the complement of $\mathcal{R}_{\lambda}$ that contain critical points. Let $U_{2}$ be the open disk containing $c_{2}$, and $U_{-1}$ the open disk containing $c_{-1}$. Then $U_{2}$ is the region that separates the full rays in $S_{2}$ from those in $S_{1}$, while $U_{-1}$ separates the full rays in $S_{-1}$ from those in $S_{-2}$. 


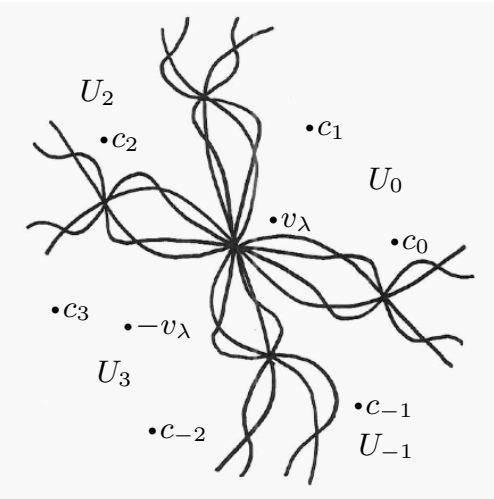

Fig. 6. Some of the rays in $\mathcal{R}_{\lambda}$ and the $U_{j}$.

Let $\mathcal{X}_{\lambda}$ denote the complement of the two disks $U_{0}$ and $U_{3}$. Then $\mathcal{X}_{\lambda}$ is a closed subset of the Riemann sphere that contains $\mathcal{R}_{\lambda}, U_{2}, U_{-1}$, and countably many other open disks.

We next discuss how $F_{\lambda}$ acts on the complementary domains in $\mathcal{R}_{\lambda}$. First, $\mathcal{R}_{\lambda}$ is $F_{\lambda}$-invariant and $F_{\lambda}$ maps this set of full rays four-to-one onto itself. It follows that the open disk $U_{2}$ is mapped two-to-one onto $U_{0}$ since $F_{\lambda}\left(c_{2}\right)=v_{\lambda}$. Similarly, $U_{-1}$ is mapped two-to-one onto $U_{3}$. These are the only open disks in $\mathcal{X}_{\lambda}$ that are mapped outside $\mathcal{X}_{\lambda}$.

There must be two other preimages of $\mathcal{X}_{\lambda}$, and so one of these preimages lies in $U_{0}$; call this preimage $\mathcal{X}_{\lambda}^{0}$. The other preimage lies in $U_{3}$ and is called $\mathcal{X}_{\lambda}^{3}=-\mathcal{X}_{\lambda}^{0}$. Note that each of these sets is mapped one-to-one onto $\mathcal{X}_{\lambda}$. Thus we have all of the preimages of $\mathcal{X}_{\lambda}$ and all we need are the four remaining preimages of $U_{0}$ and $U_{3}$. These necessarily lie in $U_{0}$ and $U_{3}$.

Since $v_{\lambda}$ lies in $U_{0}$, it follows that there is an open set in $U_{0}-\mathcal{X}_{\lambda}^{0}$ that contains $c_{0}$ and is mapped two-to-one onto $U_{0}$ and another open set that contains $c_{1}$ and is mapped two-to-one onto $U_{3}$. Similarly, there are a pair of open sets in $U_{3}-\mathcal{X}_{\lambda}^{3}$ each of which contains a critical point and is mapped two-to-one onto either $U_{0}$ or $U_{3}$. To summarize all of this, we have shown:

Proposition.

1. $F_{\lambda}$ maps $U_{2}$ two-to-one onto $U_{0}$.

2. $F_{\lambda}$ maps $U_{-1}$ two-to-one onto $U_{3}$.

3. $F_{\lambda}$ maps $U_{0}$ (and $\left.U_{3}\right)$ two-to-one onto $U_{0} \cup U_{3}$ and one-to-one onto $\mathcal{X}_{\lambda}$.

4. $F_{\lambda}$ maps $\mathcal{X}_{\lambda}$ two-to-one onto both $U_{3}$ and $U_{0}$ and four-to-one onto itself.

In order to prove the existence of baby Mandelbrot sets for this family, we first recall the theory of polynomial-like maps. Suppose $V^{\prime} \subset V$ are a pair of bounded, open, simply connected subsets of $\mathbb{C}$ with $V^{\prime}$ relatively compact 
in $V$. A map $G: V^{\prime} \rightarrow V$ is called a polynomial-like map of degree two if $G$ is holomorphic and proper of degree two. Hence such a map has a unique critical point $c \in V^{\prime}$. The filled Julia set of $G$ is defined in the natural manner as the set of points whose orbits never leave the subset $V^{\prime}$ under iteration of $G$. By the results in [7], it is known that $G$ is topologically conjugate to some quadratic polynomial in a neighborhood of the polynomial's filled Julia set in $\mathbb{C}$, hence the name polynomial-like.

Now suppose that we have a family of polynomial-like maps $G_{\mu}: V_{\mu}^{\prime} \rightarrow V_{\mu}$ depending on a parameter $\mu \in \mathbb{C}$ and satisfying:

1. The parameter $\mu$ lies in an open set in $\mathbb{C}$ that contains a closed disk $W$, and the boundaries of $V_{\mu}^{\prime}$ and $V_{\mu}$ vary analytically as $\mu$ varies.

2. The map $(\mu, z) \mapsto G_{\mu}(z)$ depends holomorphically on both $\mu$ and $z$.

3. Each $G_{\mu}: V_{\mu}^{\prime} \rightarrow V_{\mu}$ is polynomial-like of degree two.

Then we may consider the set of parameters in $W$ for which the orbit of the critical point, $c_{\mu}$, does not escape from $V_{\mu}^{\prime}$ and so the corresponding filled Julia set is connected. Suppose that for each $\mu$ in the boundary of $W$ we have that $G_{\mu}\left(c_{\mu}\right)$ lies in $V_{\mu}-V_{\mu}^{\prime}$ and that, moreover, $G_{\mu}\left(c_{\mu}\right)-c_{\mu}$ winds once around 0 as $\mu$ winds once around the boundary of $W$. Then, in this case, it is also shown in [7] that the set of $\mu$-values for which the orbit of $c_{\mu}$ does not escape from $V_{\mu}^{\prime}$ is homeomorphic to the Mandelbrot set and that the polynomial to which $G_{\mu}$ corresponds under this homeomorphism is conjugate to $G_{\mu}$ on some neighborhood of its Julia set. This result thus gives a criterion for proving the existence of small copies of a Mandelbrot set inside $\mathcal{H}$.

As a warm-up to how we will proceed to prove the existence of baby Mandelbrot sets, consider the following scenario. Recall that the region $\mathcal{X}_{\lambda}^{0}$ is mapped univalently over $\mathcal{X}_{\lambda}$. Hence there is an open disk $V_{\lambda}^{\prime} \subset \mathcal{X}_{\lambda}^{0}$ that is mapped univalently onto the region $U_{2}$. But then $U_{2}$ is mapped two-to-one onto $U_{0} \supset V_{\lambda}^{\prime}$. So it appears that $F_{\lambda}^{2}$ is a polynomial-like map taking $V_{\lambda}^{\prime}$ onto the larger set $U_{0}$. In addition, let the subset of the parameter plane we are considering be the entire region $\mathcal{H}$. Then the critical orbit for $F_{\lambda}^{2} \mid V_{\lambda}^{\prime}$ contains $c_{2}$ and lands, at the second iteration, on $v_{\lambda}$. As $\lambda$ winds once around $\mathcal{H}$, the critical value then winds once around the sector given by $0 \leq \operatorname{Arg} z \leq \pi / 2$.

There are two problems with this argument. First, the disk $V_{\lambda}^{\prime}$ actually extends all the way to $\infty$, so $V_{\lambda}^{\prime}$ is not properly contained in $F_{\lambda}^{2}\left(V_{\lambda}^{\prime}\right)$. Second, as we wind around the boundary of $\mathcal{H}$, the critical value is no longer defined for all $\lambda$.

To remedy these defects, we first restrict attention to the subset $\mathcal{S}$ of $\mathcal{H}$ given by $\mathcal{S}=\{\lambda \in \mathbb{C}|| \lambda \mid<2$ and $\operatorname{Im} \lambda>0\}$. Secondly, we constrain the open disks $U_{j}$ to lie in a region that is bounded away from both 0 and $\infty$. We accomplish this as follows. Choose a level curve $\beta_{0}$ of the Green's function lying in $B_{\lambda}$ and surrounding $J\left(F_{\lambda}\right)$. Then $F_{\lambda}$ maps $\beta_{0}$ strictly outside itself. 
Let $\beta_{1}$ be the curve in $T_{\lambda}$ that is mapped onto $F_{\lambda}\left(\beta_{0}\right)$. Let $A_{\lambda}$ denote the annulus bounded by $\beta_{0}$ and $\beta_{1}$. So we see that, if $\lambda \in \mathcal{S}, F_{\lambda}$ maps both boundary curves of $A_{\lambda}$ strictly outside the $\beta_{0}$. Furthermore, all of the critical points of $F_{\lambda}$ lie in $A_{\lambda}$.

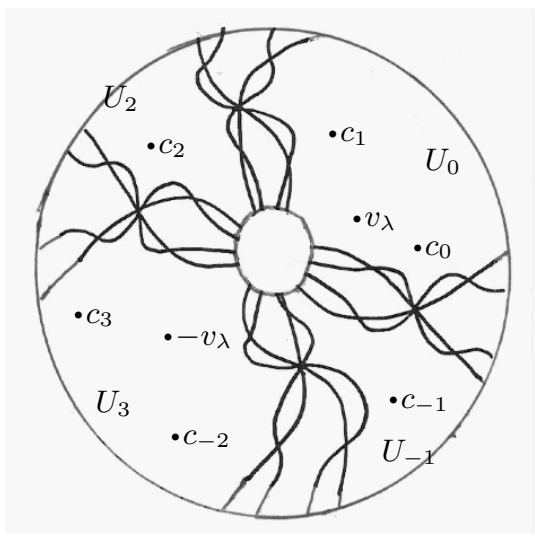

Fig. 7. The regions $\mathcal{R}_{\lambda}$ and the $U_{j}$ in $A_{\lambda}$

We now consider the restriction of the sets $\mathcal{R}_{\lambda}, \mathcal{X}_{\lambda}$, and the $U_{j}$ to the annulus $A_{\lambda}$. With a slight abuse of notation, we continue to denote these sets by $\mathcal{R}_{\lambda}, \mathcal{X}_{\lambda}$, and $U_{j}$. Since $F_{\lambda}$ takes $\mathcal{X}_{\lambda}$ four-to-one over itself (and both critical points in $\mathcal{X}_{\lambda}$ are mapped outside of $\mathcal{X}_{\lambda}$ ), it follows that there are four preimages of $U_{2}$ in $\mathcal{X}_{\lambda}$. There are then 16 open disks lying in $\mathcal{X}_{\lambda}$ whose first images under $F_{\lambda}$ also lie in $\mathcal{X}_{\lambda}$ and whose image under $F_{\lambda}^{2}$ is $U_{2}$. Continuing, there are $4^{k-2}$ open disks in $\mathcal{X}_{\lambda}$ whose images under $F_{\lambda}^{j}, j=0, \ldots, k-2$, lie in $\mathcal{X}_{\lambda}$ and such that $F_{\lambda}^{k-2}$ maps each of these sets univalently onto $U_{2}$. Hence $F_{\lambda}^{k-1}$ maps each of these sets in two-to-one fashion onto a set that contains $U_{0}$ and so the image of these sets completely contains the closure of the set $\mathcal{X}_{\lambda}^{0} \subset U_{0}$. Moreover, the critical value for $F_{\lambda}^{k-1}$ on these sets is $v_{\lambda}$.

Now $F_{\lambda}$ takes $\mathcal{X}_{\lambda}^{0}$ univalently over a region containing $\mathcal{X}_{\lambda}$. So there are $4^{k-2}$ open disks in $\mathcal{X}_{\lambda}^{0}$ each of which is mapped univalently onto one of the $4^{k-2}$ open disks in $\mathcal{X}_{\lambda}$ that are mapped by $F_{\lambda}^{k-2}$ over $U_{2}$. Hence $F_{\lambda}^{k}$ maps each of these open disks in two-to-one fashion onto a disk that properly contains each of them. Thus $F_{\lambda}^{k}$ is a polynomial-like map of degree two on each of these subsets. If we then let $\lambda$ travel around the boundary of $\mathcal{S}$, then the critical value of $F_{\lambda}^{k}$ on each of these sets, namely $v_{\lambda}$, travels once around the quarter of a disk bounded by $|z|=2 \sqrt{2}, 0 \leq \operatorname{Arg} \lambda \leq \pi / 2$, together with the intervals in the positive real and imaginary axes connecting the origin and $2 \sqrt{2}$. So the critical value winds once around each of the preimages in $\mathcal{X}_{\lambda}^{0}$. This shows that there are $4^{k-2}$ Mandelbrot sets with base period $k$ 
(i.e., the period of the cycle corresponding to parameters drawn from the main cardioid of these Mandelbrot sets is $k$ ).

REMARKS. 1. Technically, we should not move the parameter around the boundary of $\mathcal{S}$ since, when we pass through the origin, the critical value $2 \sqrt{\lambda}$ no longer varies analytically. However, if we take a slight diversion along a semi-circle surrounding the origin and lying in the McMullen domain, the previous argument then works as well. This proves the existence of infinitely many copies of the Mandelbrot set in $\mathcal{S}$.

2. For parameters drawn from these Mandelbrot sets, there are actually two attracting cycles with base period $k$. This follows since $F_{\lambda}(-z)=$ $-F_{\lambda}(z)$. In the case above, there is only one preimage of $U_{2}$ lying in $\mathcal{X}_{\lambda}^{0}$ and none in $\mathcal{X}_{\lambda}^{3}$. So the negatives of these preimages give another polynomial-like map with exactly one preimage in $\mathcal{X}_{\lambda}^{3}$ and none in $\mathcal{X}_{\lambda}^{0}$.

We call the Mandelbrot sets constructed above Type 1 Mandelbrot sets, for there is another way Mandelbrot sets arise in the parameter plane, and parameters from these Mandelbrot sets have somewhat different dynamical behavior. Consider the $4^{k-2}$ preimages of $U_{2}$ described above and lying in $\mathcal{X}$. There are the same number of preimages of these sets in $\mathcal{X}_{\lambda}^{3}$, not $\mathcal{X}_{\lambda}^{0}$ as was considered above. But then each of these sets is mapped by $F_{\lambda}^{k}$ two-to-one over the exact same region containing $U_{0}$ above. So, for definiteness, call one of these preimages $V$. Then $F_{\lambda}^{k}$ maps $V$ two-to-one over a region that properly contains the set $-V$, not $V$. Now consider the map $-F_{\lambda}^{k}$ on $V$. This map is now a polynomial-like map of degree two on $V$, and, as above, in the parameter plane corresponding to the map $-F_{\lambda}^{k}$ there is again a copy of the Mandelbrot set. But the dynamics of the second iterate of $-F_{\lambda}^{k}$ and the map of $F_{\lambda}^{2 k}$ are the same, so there is also a copy of a Mandelbrot set for our family corresponding to the same parameters. The difference here is that maps drawn from the main cardioid of this set now have an attracting cycle of period $2 k$. Also, there are two critical points lying in the immediate basin of this cycle, not 1 , and the cycle is symmetric under $z \mapsto-z$. We say that these Mandelbrot sets also have base period $k$. This produces an additional collection of $4^{k-2}$ baby Mandelbrot sets. We say that these Mandelbrot sets also have base period $k$, and we call them Type 2 baby Mandelbrot sets. We get a similar number of Type 1 and 2 Mandelbrot sets in the lower half of the parameter plane by the complex conjugation symmetry, so this gives a total of $4^{k-1}$ Type 1 and Type 2 baby Mandelbrot sets in the parameter plane. Therefore we have proved:

Theorem. Let $k \geq 2$. For the family of maps $F_{\lambda}(z)=z^{3}+\lambda / z^{3}$, there are $2 \cdot 4^{k-2}$ Type 1 baby Mandelbrot sets with base period $k$ in the parameter plane for $F_{\lambda}$ and the same number of Type 2 baby Mandelbrot sets with base period $k$. 
6. Final remarks. In this paper we have concentrated on the family of maps $z^{3}+\lambda / z^{3}$. But all of the above results (except for the piecewise linear model) go through in essentially the same manner for the families $z^{n}+\lambda / z^{n}$ with $n>3$. The difference here is that we now work with $2 n$ sectors given by $S_{0}, S_{1}, \ldots, S_{n}$ together with $S_{-j}=-S_{j}$ for $j=1, \ldots, n-1$. The parameter plane now has $(n-1)$-fold symmetry, so we restrict attention to parameters that satisfy $0<\operatorname{Arg} \lambda<2 \pi /(n-1)$. Then, as before, the two critical values $\pm 2 \sqrt{\lambda}$ lie in $S_{0}$ and $S_{n}$. We then get a Cantor set of points $\Lambda_{\lambda}$ lying in the Julia set on which $F_{\lambda}$ is conjugate to the shift map on the $2 n-2$ symbols $\pm 1, \ldots, \pm(n-1)$. Let $\Sigma$ now denote this sequence space.

Then we can define the relevant subset $\Gamma \subset \Sigma$ in two ways. First, $\Gamma$ consists of all points on the unit circle whose angles mod 1 always lie in the pair of arcs given by $[1 /(n+1), 1 / 2]$ and $[n /(n+1), 1]$. As a subshift of $\Sigma$, $\Gamma$ can also be defined as all sequences of symbols $\pm 1, \pm 2, \ldots \pm(n-1)$ where

1. odd positive integers are only followed by negative integers;

2. even positive integers are only followed by positive integers;

3. odd negative integers are only followed by positive integers when $n$ is odd, but, if $n$ is even, odd negative integers are only followed by negative integers;

4. even negative integers are only followed by negative integers when $n$ is odd, but, if $n$ is even, even negative integers are only followed by positive integers.

Then the definition of the full and internal rays goes through exactly as above.

We can also use the same techniques to produce copies of baby Mandelbrot sets as in the previous section. One difference here is that, when $n$ is even, we no longer have the $z \mapsto-z$ symmetry that creates Type 1 and 2 Mandelbrot sets of base period $k$; instead, since all of the critical points map to the same point at iteration two, the above techniques always produce attracting cycles with period $k$, never $2 k$. In any event, a similar count yields the existence of $(n-2)(2 n-2)^{k-1}$ baby Mandelbrot sets of base period $k$.

\section{References}

[1] R. L. Devaney, A myriad of Sierpiński curve Julia sets, in: Difference Equations, Special Functions and Orthogonal Polynomials, World Sci., 2007, 131-148.

[2] —, The McMullen domain: Satellite Mandelbrot sets and Sierpiński holes, Conformal Geom. Dynam. 11 (2007), 164-190.

[3] —, Baby Mandelbrot sets adorned with halos in families of rational maps, in: Complex Dynamics; Twenty-Five Years After the Appearance of the Mandelbrot Set, Contemp. Math. 396, Amer. Math. Soc., 2006, 37-50. 
[4] R. L. Devaney, Intertwined internal parameter plane rays for rational maps, to appear.

[5] -, Cantor necklaces and structurally unstable Sierpinski curve Julia sets for rational maps, Qual. Theory Dynam. Systems 5 (2004), 337-359.

[6] R. L. Devaney, D. M. Look and D. Uminsky, The escape trichotomy for singularly perturbed rational maps, Indiana Univ. Math. J. 54 (2005), 1621-1634.

[7] A. Douady and J. Hubbard, On the dynamics of polynomial-like mappings, Ann. Sci. École Norm. Sup. (4) 18 (1985), 287-343.

[8] C. McMullen, Automorphisms of rational maps, in: Holomorphic Functions and Moduli, Vol. 1, Math. Sci. Res. Inst. Publ. 10, Springer, New York, 1988, 31-60.

[9] J. Milnor, Dynamics in One Complex Variable, Vieweg, 1999.

[10] M. Misiurewicz, On iterates of $e^{z}$, Ergodic Theory Dynam. Systems 1 (1981), 103106.

[11] C. Petersen and G. Ryd, Convergence of rational rays in parameter spaces, in: The Mandelbrot Set: Theme and Variations, London Math. Soc. Lecture Note Ser. 274, Cambridge Univ. Press, 2000, 161-172.

Robert L. Devaney

Department of Mathematics

Boston University

Boston, MA 02215, U.S.A.

E-mail: bob@bu.edu

Received 22 December 2008;

in revised form 7 July 2009 